\section{Coordenação da assistência prestada às pessoas que vivem com HIV/AIDS em um município do Estado de São Paulo, Brasil}

\author{
Coordination of care for people living with \\ HIV/AIDS in a city in São Paulo State, Brazil \\ Coordinación de la atención a personas que \\ viven con $\mathrm{VIH} / \mathrm{SIDA}$ en un municipio del estado \\ de São Paulo, Brasil
}

Livia Maria Lopes 1

Gabriela Tavares Magnabosco ${ }^{1}$

Rubia Laine de Paula Andrade 1

Maria Amélia Zanon Ponce 2

Anneliese Domingues Wysocki 1

Glaucia Morandim Ravanholi 1

Jordana de Almeida Nogueira 3

Ione Carvalho Pinto 1

Lis Aparecida Neves 4

Fátima Regina de Almeida Lima Neves 5

Renata Karina Reis 1

Tereza Cristina Scatena Villa 1

Lúcia Marina Scatena 6

Aline Aparecida Monroe ${ }^{1}$

\title{
Resumo
}

1 Escola de Enfermagem de Ribeirão Preto, Universidade de São Paulo, Ribeirão Preto, Brasil.

2 Secretaria Municipal de Saúde de São José do Rio Preto, São José do Rio Preto, Brasil

3 Universidade Federal da Paraíba, João Pessoa, Brasil. 4 Programa Municipal de Controle da Tuberculose, Secretaria Municipal de Saúde, Ribeirão Preto, Brasil. 5 Programa Municipal de DST/AIDS e Hepatites Virais, Secretaria Municipal de Saúde, Ribeirão Preto, Brasil. 6 Universidade Federal do Triangulo Mineiro, Uberaba, Brasil.

Correspondência L. M. Lopes Escola de Enfermagem de Ribeirão Preto, Universidade de São Paulo.

Av. Padre Antonio Vieira 530, Franca, $S P$ 14409-212, Brasil. liviamarialopes_enf@yahoo. com.br

\begin{abstract}
The aim of this study was to analyze the coordination of health activities and services for persons living with AIDS. This was a cross-sectional study of 301 persons living with AIDS. The analysis used descriptive techniques, analysis of variance, and multiple comparisons of means. Coordination of care was found to be satisfactory (mean $=3.69$ and $S D=1.74$ ). Persons living with AIDS used other health services in addition to the Services for Specialized HIVIAIDS Care (67.0\% emergency departments, $43.2 \%$ primary care, $23.6 \%$ other specialized services, $15 \%$ private services). The five specialized HIVIAIDS clinics showed different performance levels as well as distinct contexts and healthcare configurations, but adequate clinical management (comparatively better than management of social issues). Provision of the referral guide was considered satisfactory, but the counter-referral guide was found to be unsatisfactory. Strategies are needed to promote the development of shared and cooperative actions within the healthcare teams in the specialized HIVIAIDS clinics and between the different services in order to strengthen the provision of care with case-resolution capacity.
\end{abstract}

Acquired Immunodeficiency Syndrome; Employee Performance Appraisal; Health Services; Systems Integration
Objetivou-se analisar a coordenação das ações e serviços de saúde na assistência às pessoas que vivem com HIVIAIDS. Trata-se de um estudo seccional com 301 pessoas que vivem com HIVI AIDS. A análise foi feita por técnicas descritivas, analise de variância e comparação múltiplas de médias. Encontrou-se que a coordenação da assistência foi satisfatória (média = 3,69 $e$ $D P=1,74)$. As pessoas que vivem com HIVIAIDS utilizavam outros serviços de saúde além dos Serviços de Assistência Especializada em HIVI AIDS - SAE (Pronto Atendimento: 67\%; atenção básica: 43,2\%; outros serviços especializados: 23,6\%; serviços privados: $15 \%$ ). Os cinco $S A E$ apresentaram diferentes desempenhos, bem como distintos contextos e composições assistenciais, entretanto adequado manejo dos aspectos clínicos em detrimento dos sociais. O fornecimento da guia de referência foi tido como satisfatório, entretanto a guia de contrarreferência insatisfatório. Há necessidade de estratégias que favoreçam o desenvolvimento de ações compartilhadas e cooperadas dentro das equipes dos SAE e entre os diferentes serviços com o intuito de fortalecer a produção de cuidado resolutivo.

Síndrome de Imunodeficiência Adquirida; Avaliação de Desempenho Profissional; Serviços de Saúde; Integração de Sistemas 


\section{Introdução}

Mesmo após três décadas de surgimento, a AIDS ainda é considerada um dos mais sérios problemas de saúde pública, representando um fenômeno global, dinâmico e instável, amplamente marcado pelos contrastes sociais e regionais 1 , cuja ocorrência nas diferentes regiões do mundo depende tanto do comportamento individual e coletivo ${ }^{2}$ como das dimensões política, de gestão e de organização do setor saúde.

As políticas públicas brasileiras voltadas à prevenção e controle da infecção pelo vírus da imunodeficiência humana (HIV) aliadas ao engajamento do movimento social 3 , bem como a intensa militância de profissionais de saúde e instituições de pesquisa, contribuíram para implementar a universalidade do acesso à assistência às pessoas que vivem com HIV/AIDS com distribuição gratuita desde preservativos masculinos 4 até a terapia antirretroviral (TARV). Tais avanços impactaram na morbimortalidade e qualidade de vida dos indivíduos acometidos pelo agravo 5 . Nesse sentido, a AIDS passou pela transição de enfermidade aguda e mortal para condição crônica que traz consigo importantes desdobramentos sociais, econômicos, culturais, psicológicos e, consequentemente, assistenciais 6 .

Vislumbra-se um cenário de otimismo político apontando a possibilidade do fim da epidemia, com reflexos na pactuação internacional de metas que almejam zerar o número de novas infecções pelo vírus, a discriminação pela doença, e os óbitos por HIV/AIDS 7,8. Pensar no alcance destas metas no cenário nacional, onde mais de 38 mil indivíduos foram notificados com a doença em 20119 remete a reflexões sobre a complexidade na produção do cuidado às pessoas que vivem com HIV/AIDS de modo a garantir o acesso às ações de prevenção e diagnóstico precoce e, sobretudo ao cuidado integral, coordenado e resolutivo.

As limitações assistenciais às pessoas que vivem com HIV/AIDS decorrem do trabalho fragmentado da equipe de saúde e das fragilidades nos mecanismos de referência e contrarreferência como parte do processo de integração e coordenação das ações ofertadas dentro de um mesmo serviço e entre diferentes pontos de atenção à saúde 10,11, sendo essa condição sine qua non para a constituição e efetivação de uma rede de atenção à saúde para o manejo do agravo.

Neste contexto, a coordenação da assistência às pessoas que vivem com HIV/AIDS parte da elaboração de um plano de cuidados compartilhado, implicando a sistematização e integração de uma prática interdisciplinar e intersetorial, a organização do processo de trabalho e a in- corporação de novos saberes e práticas 12,13,14. Concebe-se, assim, a necessidade de atribuir responsabilidades entre os diversos serviços e profissionais de saúde em consonância com a densidade tecnológica e o conhecimento disponível, devendo os pontos de atenção cooperar entre si para atender as reais demandas de determinada população 15,16 .

Reconhece-se assim que, apesar da evolução das políticas de saúde para as pessoas que vivem com HIV/AIDS e avanços à terapêutica do agravo, o manejo da assistência está passível às fragilidades relacionadas à coordenação das ações e serviços de saúde. Nesse sentido, esse estudo, destaca-se pela aproximação e parceria entre academia e serviços de saúde, a utilização do método científico tendo como sujeito o usuário na abordagem quantitativa, a imersão no cenário de prática para compreensão do contexto que permeia a organização e a assistência prestada no "micro espaço", e diferencia-se da maioria dos outros estudos que estão centrados na avaliação da assistência sob a percepção dos profissionais e/ou gestores dos serviços de saúde 10,12,17,18,19.

Diante do exposto, este estudo objetivou analisar a coordenação das ações e serviços de saúde na assistência prestada às pessoas que vivem com HIV/AIDS em um município de grande porte do interior paulista.

Para isto, o estudo se fundamentou no conceito de coordenação proposto por Starfield 20, caracterizado como um atributo da atenção primária à saúde que possibilita a produção e continuidade do cuidado, por meio do reconhecimento de problemas/demandas de saúde anteriores e/ou novas, incluindo o encaminhamento e acompanhamento do atendimento em outros serviços especializados. Adicionalmente, assume-se que o conceito de coordenação possui duas dimensões essenciais: integração vertical que ocorre entre diferentes pontos e setores de atenção à saúde dentro de uma rede assistencial; horizontal, entre membros de uma mesma equipe 21. Partindo-se da premissa de que a assistência às pessoas que vivem com HIV/AIDS é de responsabilidade dos Serviços de Assistência Especializada em HIV/AIDS (SAE), reconhece-se que a coordenação pode também ser considerada um atributo de tais serviços na medida em que tal agravo constitui uma condição crônica que requer acompanhamento ao longo do tempo.

\section{Métodos}

Trata-se de um estudo seccional, do tipo inquérito exploratório, realizado no Município de Ribeirão Preto, São Paulo, Brasil, cuja população 
estimada em 2011 era de 614.759 habitantes (Fundação Sistema Estadual de Análise de Dados. População e Estatísticas Vitais: Perfil Municipal. http://www.seade.gov.br/produtos/perfil/ perfilMunEstado.php, acessado em 20/Jul/2012). Nesse mesmo ano, a rede pública de atenção à saúde no município se organizava em cinco Distritos Sanitários, do qual faziam parte unidades de pronto atendimento, serviços de atenção primária (unidades de atenção básica), secundária (onde se inseriam cinco SAE que integravam o Programa de DST/AIDS) e terciária.

O acompanhamento clínico-terapêutico dos casos de HIV/AIDS diagnosticados e notificados no município era realizado por equipes especializadas dos SAE, designados com as letras "A", "B", "C", "D" e "E" no intuito de preservar a identidade e diferenciá-los, uma vez que possuem formas de organização distintas. Destaca-se que os SAE contam com a retaguarda de um Hospital Universitário de grande porte para atendimento aos casos graves que necessitavam de internação (Secretaria Municipal de Saúde, Prefeitura Municipal de Ribeirão Preto. Aconselhamento em DST/AIDS e Hepatites Virais. http://www.saude. ribeiraopreto.sp.gov.br/ssaude/programas/aids/ i16indice.php, acessado em 17/Fev/2012).

\section{População de estudo e amostra}

A população do estudo foi constituída pelas pessoas que vivem com HIV/AIDS em acompanhamento nos referidos SAE que atendiam aos critérios de inclusão: casos confirmados da doença, com idade igual ou superior a 18 anos, TARV instituída há mais de seis meses, residentes no município de estudo e não pertencentes ao sistema prisional.

O cálculo para o tamanho da amostra foi realizado a partir do levantamento do número total de casos confirmados e acompanhados pelos cinco ambulatórios em janeiro/2011, sendo identificados 1.389 casos (249 pertencentes ao ambulatório C, 249 ao A; 374 ao D; 374 ao B e 143 ao E). Os parâmetros considerados foram: erro amostral de $5 \%(e=0,05)$; intervalo de $95 \%$ de confiança $(Z=1,96)$ e proporção populacional (P) de $50 \%$. Obteve-se por meio da equação $n_{0}=P \cdot(1-P) \cdot Z^{2} / e^{2}$ a amostra mínima de 385 de indivíduos. Este valor foi corrigido em relação à população total, resultando em 301 indivíduos a serem abordados no estudo, considerando-se uma perda potencial de $5 \%$.

Realizou-se o processo de amostragem em duas etapas: estratificação com partilha proporcional segundo o SAE que acompanhava o caso e amostragem por conveniência (considerando as oportunidades de comparecimento das pessoas que vivem com HIV/AIDS aos serviços, a cada dois/três meses e as questões de confidencialidade e privacidade do sujeito) até atingir o tamanho da amostra. Assim, levando-se em consideração o número de indivíduos previsto em cada SAE, 54 deveriam ser entrevistados no ambulatório A; 81 no B; 54 no C; 81 no D; e 31 no E.

\section{Coleta de dados e instrumento estruturado}

Para coleta de dados realizaram-se entrevistas utilizando um questionário estruturado elaborado a partir de guideline 22 , protocolo 23 e artigo que aborda avaliação organizacional de serviços de saúde 24. Tal instrumento foi submetido à análise de conteúdo e semântica visando ajustes e adequações necessárias 25. Para este estudo, foram selecionadas variáveis relacionadas às características sociodemográficas e clínicas de acompanhamento das pessoas que vivem com HIV/AIDS, serviço de saúde utilizado e coordenação da assistência. Esta última foi constituída pela "coordenação da assistência no SAE" e "coordenação da assistência junto a outros serviços” e essas são as variáveis de interesse central deste estudo. As categorias de respostas das variáveis selecionadas contemplavam escalas dicotômicas, múltipla escolha e Likert, com valor entre "um" e "cinco", cuja resposta mais favorável correspondia ao valor mais alto (Tabela 1 ).

A coleta de dados foi realizada por cinco entrevistadores treinados entre julho de 2011 a fevereiro de 2012, em áreas que resguardassem a privacidade dos entrevistados, considerando as seguintes oportunidades de comparecimento aos serviços: antes ou após as consultas médicas/ de enfermagem, após a retirada da TARV e coleta de material para realização de exames (contagem de linfócitos T-CD4+/CD8 e carga viral).

Ressalta-se que para a caracterização dos locais de estudo e identificação dos elementos subjetivos relacionados ao contexto organizacional e político utilizou-se roteiro observacional elaborado com base no Qualiaids 26 .

\section{Análise de dados}

As variáveis sociodemográficas e de acompanhamento clínico foram analisadas por distribuição de frequência, média e desvio-padrão. Para analisar a coordenação da assistência às pessoas que vivem com HIV/AIDS foram construídos indicadores que corresponderam à somatória das categorias das respostas das pessoas que vivem com HIV/AIDS de cada variável dividido pelo total de pessoas que vivem com HIV/AIDS para obtenção de um valor médio. Foram construídos indicadores compostos, que corresponderam ao 
Tabela 1

Variáveis utilizadas no estudo segundo a seção do instrumento de coleta de dados e categorias de respostas.

\begin{tabular}{|c|c|}
\hline Seção/Variáveis & Categorias de respostas \\
\hline \multicolumn{2}{|l|}{ Características sociodemograficas } \\
\hline Sexo & () Feminimo () Masculino \\
\hline Idade & _ anos \\
\hline \multirow[t]{5}{*}{ Escolaridade } & () Sem escolaridade ou Ensino Fundamental \\
\hline & incompleto; \\
\hline & () Ensino Fundamental completo; \\
\hline & () Ensino Médio completo; \\
\hline & () Ensino Superior completo \\
\hline \multirow[t]{4}{*}{ Situação empregatícia } & () Empregado; ( ) Autônomo; \\
\hline & () Aposentado; () Pensionista; \\
\hline & () Desempregado; () Afastado; \\
\hline & () Estudante; () Do lar \\
\hline Classificação econômica * & () $A() B() C() D() E$ \\
\hline \multicolumn{2}{|l|}{ Características clínicas de acompanhamento do caso } \\
\hline Tempo de tratamento do HIV/AIDS & __ meses \\
\hline Status clínico & () Sintomático () Assintomático \\
\hline Linfócitos T-CD4+ & ()$<350($ () 350-499() $>500$ \\
\hline Carga viral & () Indetectável () Detectável \\
\hline Condições crônicas associadas & () Sim () Não \\
\hline \multicolumn{2}{|l|}{ Coordenação da assistência no SAE } \\
\hline V1 - Leitura o prontuário/ficha durante a consulta & () $1 \quad() 2 \quad() 3 \quad() 4 \quad() 5$ \\
\hline V2 - Anotação no prontuário durante a consulta médica/enfermagem & () 1 () 2 () 3 () 4 () 5 \\
\hline V3 - Recebimento dos resultados impressos dos exames & () $1 \quad() 2 \quad() 3 \quad() 4 \quad() 5$ \\
\hline V4 - Resultados de exames prontos e disponíveis quando comparece ao serviço & () $1 \quad() 2 \quad() 3 \quad() 4 \quad() 5$ \\
\hline V5 - Avisado sobre o agendamento da consulta de retorno & () $1 \quad() 2 \quad() 3 \quad() 4 \quad() 5$ \\
\hline V6 - Questionamento sobre a regularidade no uso dos medicamentos & () $1 \quad() 2 \quad() 3 \quad() 4 \quad() 5$ \\
\hline V7 - Questionamento sobre a ocorrência de efeitos colaterais & () $1 \quad() 2 \quad() 3 \quad() 4 \quad() 5$ \\
\hline V8 - Questionamento sobre aparecimento de intercorrências & () $1 \quad() 2$ () 3 () 4 () 5 \\
\hline V9 - Questionamento sobre as condições de vida das pessoas que vivem com HIV/AIDS & () 1 () 2 () 3 () 4 () 5 \\
\hline V10 - Questionamento sobre o recebimento de apoio durante o tratamento & () $1 \quad() 2 \quad() 3 \quad() 4 \quad() 5$ \\
\hline V11 - Orientação sobre ONGs & () $1 \quad() 2 \quad() 3 \quad() 4 \quad() 5$ \\
\hline V12 - Utilização da atenção básica & () $1 \quad() 2 \quad() 3 \quad() 4 \quad() 5$ \\
\hline V13 - Utilização de Pronto Atendimento & () $1 \quad() 2 \quad() 3 \quad() 4 \quad() 5$ \\
\hline V14 - Utilização de outros ambulatórios públicos & () $1 \quad() 2 \quad() 3 \quad() 4 \quad() 5$ \\
\hline \multicolumn{2}{|l|}{ Coordenação da assistência entre outros serviços } \\
\hline V15 - Encaminhamento para atendimento com outros profissionais e serviços de saúde & () 1 () 2 () 3 () 4 () 5 \\
\hline V16 - Discussão sobre os possíveis locais de atendimento em caso de encaminhamento & () $1 \quad() 2 \quad() 3 \quad() 4 \quad() 5$ \\
\hline V17 - Auxilio para o agendamento de consultas junto a outros serviços de saúde & () $1 \quad() 2 \quad() 3 \quad() 4 \quad() 5$ \\
\hline V18 - Recebimento de comprovante do agendamento de consulta em outro serviço de saúde & () $1 \quad() 2 \quad() 3 \quad() 4 \quad() 5$ \\
\hline V19 - Fornecimento de informações escritas para ser entregue no outro serviço de saúde & () $1 \quad() 2 \quad() 3 \quad() 4 \quad() 5$ \\
\hline V20 - Obtenção de atendimento quando encaminhado & () $1 \quad() 2 \quad() 3 \quad() 4 \quad() 5$ \\
\hline V21 - Recebimento de guia de contrarreferência & () $1 \quad() 2 \quad() 3 \quad() 4 \quad() 5$ \\
\hline V22 - Discussão sobre os resultados da consulta em outro serviço & () $1 \quad() 2 \quad() 3 \quad() 4 \quad() 5$ \\
\hline V23 - Interesse quanto ao atendimento prestado em outros serviços & () 1 () 2 () 3 () 4 () 5 \\
\hline
\end{tabular}

SAE: Serviços de Assistência Especializada em HIV/AIDS.

* Classificação econômica com base na Associação Brasileira de Empresas de Pesquisa (ABEP. Critério de Classificação Econômica Brasil. http://www.abep. org/new/Servicos/Download. aspx?id=04, acessado em Mar/2011).

Nota: para as categorias de respostas para as variáveis V1 a V23 foi utilizada a escala de Likert, com valor entre 1 e 5, cuja resposta correspondia 1 = nunca; 2 = quase nunca; 3 = às vezes; $4=$ quase sempre; $5=$ sempre. 
valor médio de cada variável e de cada serviço de saúde e para o município. Os indicadores foram submetidos à análise de variância e teste de comparação múltipla de Tukey quando atendidos os pressupostos de homocedasticidade pelo teste de Levene e normalidade pelo teste de Kolmogorov-Smirnov com correção para Lilliefors. Para as análises que indicaram violação dos critérios para o uso da Anova foi usada análise de variância não-paramétrica, com o uso do teste de KruskallWallis e teste de comparação múltipla de média das ordens. O nível de significância estatística adotado em todos os testes foi de $5 \% 27$.

Os indicadores foram classificados como insatisfatórios (entre 1 e 2,5 correspondendo a valores até $50 \%$ da escala), regulares (maior que 2,5 e menor que 3,5, correspondendo a valores entre $50 \%$ e $70 \%$ da escala) e satisfatórios (maior que 3,5 , correspondendo a valores maior que $70 \%$ da escala).
Atendendo à Resolução no 196/96 do Conselho Nacional de Saúde, este estudo possui aprovação do Comitê de Ética em Pesquisa da Escola de Enfermagem de Ribeirão Preto da Universidade de São Paulo, conforme protocolo no 1.215/2010.

\section{Resultados}

Foram entrevistadas 301 pessoas que vivem com HIV/AIDS, correspondendo a $21,7 \%$ de todos os casos identificados nos registros dos SAE. Dessas, a proporção foi semelhante entre os sexos, com razão de 1,05 homens para cada mulher. A idade média foi de 45,2 anos (DP $=10,1$ ), com faixa etária predominante de 40 a 64 anos $(64,8 \%)$. A maioria apresentava baixa escolaridade $(52,8 \%)$, fonte de renda $(54,8 \%)$ e classe econômica C $(56,1 \%)$ (Tabela 2$)$.

Tabela 2

Distribuição de frequência das características sociodemográficas das pessoas que viviam com HIV/AIDS no Município de Ribeirão Preto, São Paulo, Brasil, 2011

\begin{tabular}{|c|c|c|}
\hline \multirow[t]{2}{*}{ Características sociodemográficas } & \multicolumn{2}{|c|}{ Doentes $(\mathrm{N}=301)$} \\
\hline & $\mathbf{n}$ & $\%$ \\
\hline \multicolumn{3}{|l|}{ Sexo } \\
\hline Masculino & 154 & 51,2 \\
\hline Feminino & 147 & 48,8 \\
\hline \multicolumn{3}{|l|}{ Faixa etária (anos) } \\
\hline $24-39$ & 94 & 31,2 \\
\hline $40-64$ & 195 & 64,8 \\
\hline $65-75$ & 12 & 4,0 \\
\hline \multicolumn{3}{|l|}{ Escolaridade } \\
\hline Sem escolaridade ou Ensino Fundamental incompleto & 159 & 52,8 \\
\hline Ensino Fundamental completo & 64 & 21,3 \\
\hline Ensino Médio completo & 67 & 22,3 \\
\hline Ensino Superior completo & 11 & 3,7 \\
\hline \multicolumn{3}{|l|}{ Situação empregatícia } \\
\hline Empregado/Autônomo & 165 & 54,8 \\
\hline Aposentado/Pensionista & 59 & 19,6 \\
\hline Desempregado & 46 & 15,3 \\
\hline Afastado & 13 & 4,3 \\
\hline Outros (estudante/do lar) & 18 & 6,0 \\
\hline \multicolumn{3}{|l|}{ Classificação econômica * } \\
\hline A & 1 & 0,3 \\
\hline B & 72 & 24,0 \\
\hline C & 169 & 56,1 \\
\hline D & 55 & 18,3 \\
\hline$E$ & 4 & 1,3 \\
\hline
\end{tabular}

* Classificação econômica com base na Associação Brasileira de Empresas de Pesquisa (ABEP. Critério de Classificação Econômica Brasil. http://www.abep.org/new/Servicos/Download.aspx?id=04, acessado em Mar/2011). 
O tempo médio de tratamento antirretroviral entre os entrevistados foi de 7,6 anos (DP $=4,1$ ), sendo o tempo mínimo de seis meses e máximo de vinte anos.

Quanto ao status clínico e imunológico, a maioria apresentava-se assintomático $(78,4 \%)$, com contagem de linfócitos T-CD4 acima de 500 $(61,5 \%)$ e carga viral indetectável $(76,4 \%)$. Encontrou-se que pouco mais da metade dos entrevistados $(50,2 \%)$ apresentava pelo menos alguma condição crônica associada (Tabela 3).

Quanto à utilização dos serviços de saúde, 85\% fazia uso exclusivo dos serviços públicos sendo que desses, de forma complementar à assistência do SAE, $67 \%$ utilizavam serviços de Pronto Atendimento, 43,2\% serviços da atenção básica e 23,6\% outros serviços especializados.

A coordenação da assistência às pessoas que vivem com HIV/AIDS no Município de Ribeirão Preto foi classificada como satisfatória (indicador composto - média $=3,69$; $\mathrm{DP}=1,74$ ). Em relação à "coordenação da assistência no SAE" foram considerados insatisfatórios os indicado- res "entrega dos resultados de exames impressos", "questionamento sobre condições de vida", "questionamento sobre o recebimento de apoio familiar/parceiros/amigos para o tratamento", "orientações sobre as ONGs HIV/AIDS do município", "utilização da atenção básica”, "utilização de pronto atendimento", "utilização de outros ambulatórios públicos" e os demais indicadores como satisfatórios com valores médios entre 4,23 e 4,98 (Tabela 4).

Com relação à "coordenação da assistência às pessoas que vivem com HIV/AIDS” nos serviços de saúde as principais diferenças encontradas foram: melhor desempenho para os serviços B e E (regular) em "entrega dos resultados de exames impressos"; melhor avaliação para o serviço D (regular) no "questionamento sobre condições de vida"; melhor desempenho para os serviços A, B e D (regular) quanto ao "questionamento sobre o recebimento de apoio familiar/parceiros/ amigos para o tratamento" e o serviço E (regular) com melhor avaliação quanto à "utilização da atenção básica” (Tabela 4).

Tabela 3

Distribuição de frequência das variáveis clínicas sobre o acompanhamento do caso das pessoas que viviam com HIV/AIDS no Município de Ribeirão Preto, São Paulo, Brasil, 2011.

\begin{tabular}{|c|c|c|}
\hline \multirow{2}{*}{$\begin{array}{l}\text { Variáveis clínicas sobre o } \\
\text { acompanhamento do caso }\end{array}$} & \multicolumn{2}{|c|}{ Doentes $(N=301)$} \\
\hline & $\mathrm{n}$ & $\%$ \\
\hline \multicolumn{3}{|l|}{ Status clínico } \\
\hline Assintomático & 236 & 78,4 \\
\hline Sintomático & 65 & 21,6 \\
\hline \multicolumn{3}{|l|}{ Linfócitos T-CD4+ } \\
\hline$<350$ & 67 & 22,3 \\
\hline $350-499$ & 43 & 14,2 \\
\hline$>500$ & 185 & 61,5 \\
\hline Sem informação & 6 & 2,0 \\
\hline \multicolumn{3}{|l|}{ Carga viral } \\
\hline Indetectável & 230 & 76,4 \\
\hline Detectável & 64 & 21,3 \\
\hline Sem informação & 7 & 2,3 \\
\hline \multicolumn{3}{|c|}{ Condições crônicas associadas * } \\
\hline Hipertensão arterial & 70 & 23,3 \\
\hline Depressão & 44 & 14,6 \\
\hline Dislipidemia & 30 & 10,0 \\
\hline Hepatites virais & 29 & 9,6 \\
\hline Diabetes mellitus & 23 & 7,6 \\
\hline Outros & 19 & 6,3 \\
\hline Nenhuma & 150 & 49,8 \\
\hline
\end{tabular}

* Alguns entrevistados $(n=51)$ referiram mais de uma condição crônica associada e por esse motivo a soma ultrapassou os 301 indivíduos do estudo. 
Médias e desvio-padrão (DP) dos indicadores relacionados à coordenação da assistência nos serviços de saúde no Município de Ribeirão Preto, São Paulo, Brasil, 2011.

\begin{tabular}{|c|c|c|c|c|c|c|c|}
\hline \multirow[t]{3}{*}{ Itens } & \multirow[t]{3}{*}{$\mathbf{n}$} & \multicolumn{5}{|c|}{ Serviços de saúde } & \multirow[t]{3}{*}{ Município } \\
\hline & & A & B & $\mathrm{C}$ & D & $E$ & \\
\hline & & Média \pm DP & Média \pm DP & Média \pm DP & Média \pm DP & Média \pm DP & \\
\hline 1 & 301 & $4,98 \pm 0,13$ & $4,98 \pm 0,14$ & $4,90 \pm 0,62$ & $4,96 \pm 0,19$ & $4,97 \pm 0,19$ & $4,95 \pm 0,35$ \\
\hline 2 & 300 & $5,00 \pm 0,00$ & $4,98 \pm 0,14$ & $5,00 \pm 0,00$ & $4,94 \pm 0,46$ & $5,00 \pm 0,00$ & $4,98 \pm 0,24$ \\
\hline 3 & 301 & $1,80 \pm 1,57 b$ & $2,30 \pm 1,81 \mathrm{ab}$ & $1,98 \pm 1,60 b$ & $1,74 \pm 1,38 b$ & $3,38 \pm 1,86 a$ & $2,07 \pm 1,67$ \\
\hline 4 & 301 & $4,96 \pm 0,19$ & $4,94 \pm 0,23$ & $4,76 \pm 0,56$ & $4,86 \pm 0,44$ & $4,76 \pm 0,95$ & $4,86 \pm 0,49$ \\
\hline 5 & 301 & $4,98 \pm 0,13$ & $5,00 \pm 0,00$ & $4,91 \pm 0,49$ & $4,87 \pm 0,64$ & $4,76 \pm 0,83$ & $4,92 \pm 0,49$ \\
\hline 6 & 301 & $4,98 \pm 0,13$ & $4,89 \pm 0,57$ & $4,84 \pm 0,46$ & $4,95 \pm 0,45$ & $4,86 \pm 0,74$ & $4,91 \pm 0,47$ \\
\hline 7 & 301 & $4,03 \pm 1,62$ & $4,41 \pm 1,25$ & $4,27 \pm 1,33$ & $4,26 \pm 1,45$ & $4,14 \pm 1,38$ & $4,24 \pm 1,41$ \\
\hline 8 & 301 & $3,91 \pm 1,70$ & $4,37 \pm 1,36$ & $4,46 \pm 1,24$ & $4,35 \pm 1,35$ & $4,55 \pm 1,12$ & $4,32 \pm 1,38$ \\
\hline 9 & 301 & $2,30 \pm 1,78 b c$ & $2,48 \pm 1,70 \mathrm{ab}$ & $1,42 \pm 1,03 c$ & $3,35 \pm 1,83 a$ & $2,24 \pm 1,70 \mathrm{abc}$ & $2,37 \pm 1,74$ \\
\hline 10 & 301 & $2,80 \pm 1,81 a$ & $2,61 \pm 1,80^{a}$ & $1,66 \pm 1,27 b$ & $2,97 \pm 1,84 a$ & $2,31 \pm 1,75 \mathrm{ab}$ & $2,45 \pm 1,75$ \\
\hline 11 & 189 & $1,63 \pm 1,41$ & $1,63 \pm 1,42$ & $1,52 \pm 1,16$ & $1,48 \pm 1,16$ & $1,14 \pm 0,74$ & $1,50 \pm 1,24$ \\
\hline 12 & 301 & $1,40 \pm 0,95 c$ & $2,37 \pm 1,44 a b$ & $1,86 \pm 1,30 b c$ & $1,76 \pm 1,16 b c$ & $3,34 \pm 1,54 a$ & $1,98 \pm 1,36$ \\
\hline 13 & 301 & $2,41 \pm 1,26$ & $2,42 \pm 1,11$ & $2,20 \pm 1,03$ & $2,13 \pm 1,21$ & $2,62 \pm 1,45$ & $2,31 \pm 1,19$ \\
\hline 14 & 297 & $1,44 \pm 1,13$ & $2,02 \pm 1,63$ & $1,96 \pm 1,27$ & $1,59 \pm 1,28$ & $2,00 \pm 1,41$ & $1,78 \pm 1,35$ \\
\hline 15 & 296 & $2,82 \pm 1,96 b$ & $4,22 \pm 1,45 a$ & $3,57 \pm 1,85 a$ & $3,66 \pm 1,83 a$ & $3,76 \pm 1,79 a$ & $3,59 \pm 1,83$ \\
\hline 16 & 192 & $1,64 \pm 1,44 c$ & $1,85 \pm 1,61 c$ & $3,05 \pm 1,62 \mathrm{ab}$ & $2,18 \pm 1,77 b c$ & $3,42 \pm 1,95 a$ & $2,33 \pm 1,76$ \\
\hline 17 & 199 & $4,81 \pm 0,78$ & $4,72 \pm 0,90$ & $4,20 \pm 1,17$ & $4,70 \pm 0,95$ & $4,58 \pm 1,26$ & $4,58 \pm 1,03$ \\
\hline 18 & 197 & $4,96 \pm 0,20$ & $4,87 \pm 0,53$ & $4,50 \pm 0,84$ & $4,71 \pm 1,05$ & $4,11 \pm 1,71$ & $4,68 \pm 0,93$ \\
\hline 19 & 195 & $4,65 \pm 0,93 a$ & $4,47 \pm 1,28 a$ & $2,55 \pm 1,65^{b}$ & $4,43 \pm 1,32^{a}$ & $4,53 \pm 1,33 a$ & $4,01 \pm 1,59$ \\
\hline 20 & 196 & $4,70 \pm 0,60$ & $4,72 \pm 0,85$ & $4,79 \pm 0,70$ & $4,77 \pm 0,91$ & $4,70 \pm 0,98$ & $4,75 \pm 0,81$ \\
\hline 21 & 183 & $2,00 \pm 1,76$ & $2,60 \pm 1,92$ & $1,91 \pm 1,48$ & $2,43 \pm 1,82$ & $3,00 \pm 2,06$ & $2,33 \pm 1,80$ \\
\hline 22 & 185 & $3,92 \pm 1,60 a$ & $4,20 \pm 1,54 a$ & $2,12 \pm 1,60 b$ & $3,24 \pm 1,90 a$ & $4,87 \pm 0,34 a$ & $3,42 \pm 1,83$ \\
\hline 23 & 188 & $4,00 \pm 1,55 a$ & $4,66 \pm 0,95 a$ & $3,37 \pm 1,27 b$ & $4,35 \pm 1,37 a$ & $5,00 \pm 0,00 a$ & $4,18 \pm 1,33$ \\
\hline Total & & $3,43 \pm 1,86$ & $3,73 \pm 1,74$ & $3,36 \pm 1,77$ & $3,61 \pm 1,79$ & $3,76 \pm 1,73$ & $3,69 \pm 1,74$ \\
\hline
\end{tabular}

$\mathrm{n}$ : número de respondentes;

Notas: médias seguidas pela mesma letra minúscula na linha não diferem entre si para o teste de comparação múltipla de média das ordens. Os itens correspondem às variáveis de 1 a 23 descritas na Tabela 1. Letras diferentes indicam médias diferentes. Perguntas com filtro, por isso " $n$ " diferentes para as questões.

Na "coordenação da assistência entre outros serviços" no município foram considerados insatisfatórios os indicadores "discussão sobre os possíveis locais de atendimento em caso de encaminhamento"; "recebimento de guia de contrarreferência"; regular o indicador "discussão sobre os resultados da consulta em outro serviço" e os demais indicadores foram considerados satisfatórios com valores médios entre 3,59 e 4,68 (Tabela 4).

As principais diferenças encontradas, na "coordenação da assistência entre outros serviços" em relação aos serviços de saúde foram: "encaminhamento para atendimento com outros profissionais e serviços de saúde" com o serviço A sendo avaliado como regular e os demais satisfa- tórios; serviços A, B e D insatisfatórios para "discussão sobre possíveis locais de atendimento em caso de encaminhamento" e os demais regulares; serviço $\mathrm{C}$ regular para o indicador "fornecimento de informações escritas para ser entregue no outro serviço de saúde" e insatisfatório para o indicador "discussão sobre os resultados da consulta prestada em outros serviços” (Tabela 4).

A Figura 1 apresenta um resumo dos indicadores insatisfatórios segundo o serviço de saúde utilizado. 


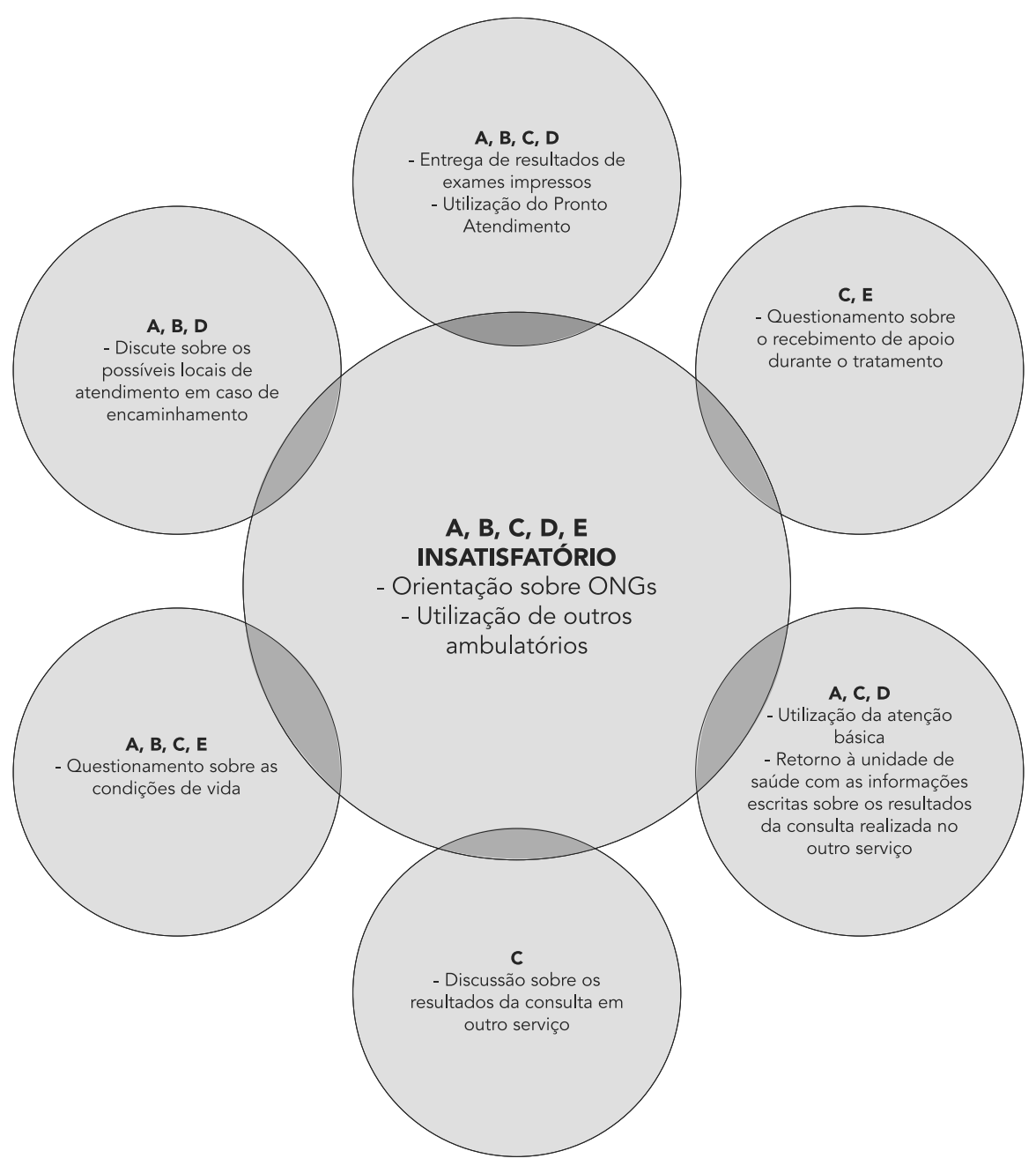

\section{Discussão}

O perfil sociodemográfico encontrado entre as pessoas que vivem com HIV/AIDS entrevistadas corrobora os achados da literatura, que evidenciam paridade da infecção entre os sexos, envelhecimento da população, baixa escolaridade, acesso a alguma modalidade de fonte de renda e predomínio da classe econômica C 28,29,30,31,32,33.

Quanto aos achados clínicos relacionados ao agravo, o tempo médio de tratamento foi de sete anos, com predomínio de sujeitos assintomáticos, carga viral indetectável e linfócitos T-CD4+ acima de 500 células $/ \mathrm{mm}^{3}$. Tais dados refletem bons níveis de adesão ao tratamento e estado imunológico ${ }^{34}$, podendo relacionar-se a um adequado desempenho dos SAE na coordenação da assistência prestada às pessoas que vivem com HIV/AIDS.

Por outro lado, o indicativo da presença de comorbidades crônicas associadas e a realização de outros tratamentos além da TARV exigem estratégias de integração entre equipes e serviços de saúde envolvidos visando o compartilhamento de informações e a co-responsabilização no processo de acompanhamento dos usuários. Entretanto, a formação profissional - culturalmente biologicista e centrada na doença - pode limitar a abordagem do especialista às dimensões clínicas em detrimento dos aspectos 
sociais que poderiam ser explorados e incorporados ao cuidado.

Assim, frente à complexidade da atenção integral, conhecer o perfil sociodemográfico e clínico, bem como de utilização de serviços permite analisar não apenas a efetividade do acesso pelos usuários, mas também a qualidade da assistência prestada, a equidade em saúde e a integralidade e resolutividade do cuidado 35,36.

Neste estudo, observou-se a utilização de outros serviços da rede pública municipal de saúde pelos usuários além dos próprios SAE, dentre eles o Pronto Atendimento e atenção básica.

Considerando o HIV/AIDS como uma condição crônica, a retaguarda assistencial de serviços de urgência e emergência como constituinte da rede de atenção à saúde 36 justifica-se pelas intercorrências no período interconsulta dos SAE (2 a 3 meses), como momentos de agudização e exacerbações clínicas relacionadas à doença e/ ou TARV. Além disso, a utilização do Pronto Atendimento pode decorrer pela expectativa de obtenção de ajuda e resolução do problema no serviço mais próximo ${ }^{14}$, com ausência de limitação quanto ao horário de atendimento, condutas de resolução em curto prazo 37 , a valorização desta atenção pelos gestores de saúde e população como reflexo do processo de transferência de políticas e incentivos financeiros 38 .

Entretanto, apesar da busca e utilização de diferentes serviços corroborar a autonomia dos usuários na escolha do local de atendimento 14 e o grau de satisfação em relação às experiências anteriores 20, sua utilização recorrente pode indicar fragilidades no acesso, vínculo, manejo da doença e, consequentemente, na coordenação da assistência prestada pelos serviços que acompanham os casos. Assim, diferentes trajetórias pelos serviços para o manejo momentâneo da condição clínica podem minimizar a suspeição da doença pela equipe e resguardar o diagnóstico 38 .

Em relação à procura por serviços da atenção básica, esta ocorreu para atendimentos médicos eventuais, retirada de medicamentos/preservativos e vacinação, além de acompanhamento ginecológico e clínico geral.

Vislumbra-se um possível processo de descentralização do atendimento edificado como competência exclusiva dos SAE, reforçado na atual conjuntura das políticas da atenção básica brasileira 11,14. No entanto, conflitos ideológicos, culturais e organizacionais (referência e contrarreferência) devem ser considerados quando se cogita a possibilidade de integrar a assistência ao HIV/AIDS em outros serviços de saúde 39 , além da disponibilidade de recursos humanos preparados, sensibilizados e motivados para uma abordagem individual 18,39.
Dessa forma, pensar na efetiva incorporação da atenção básica na produção de um cuidado coadjuvante, compartilhado e resolutivo, implica superar os desafios que esbarram em seu fortalecimento como prioridade político-assistencial, efetivando-a como porta de entrada capaz de assumir a coordenação e continuidade da assistência 40,41 .

A apropriação, o fortalecimento e a efetiva utilização dos dispositivos de apoio à integração entre os serviços são essenciais, dentre eles o próprio sistema formal de referência/contrarreferência e também o sistema informatizado online presente no município, Sistema Hygia (http:/ / www.techne.com.br/modulos.asp?id=185, com login e senha disponibilizados pela Prefeitura Municipal de Ribeirão Preto), que interliga os pontos de atenção da rede pública e fornece dados relevantes sobre os usuários que poderiam ser incluídos no delineamento e operacionalização do plano de cuidados às pessoas que vivem com HIV/AIDS.

A utilização de serviços privados nesse estudo ocorreu por meio de adesão a seguros e planos de saúde e pagamento/desembolso direto pela assistência, mediante benefícios médicos concedidos pela adesão a convênios com empresas funerárias, principalmente entre os usuários provenientes das regiões mais carentes do município. Por um lado, este acesso constitui-se como opção aos trâmites e morosidade que caracterizam a regulação do acesso à rede pública de saúde a outras especialidades 14 , por outro, pode cristalizar a fragmentação do cuidado às pessoas que vivem com HIV/AIDS.

Há ainda outra realidade assistencial viabilizada pelo princípio dauniversalidade do acesso 42 , na qual indivíduos são acompanhados pelas redes privadas de saúde e fazem uso dos SAE - de modo complementar, apenas para a retirada da TARV, distribuídos e controlados pela rede pública e, eventualmente, para a realização de exames específicos relacionados ao controle da doença.

Perante esta pluralidade e complexidade assistencial, atrelada às múltiplas facetas e repercussões biopsicossociais da doença, os novos avanços exigidos na produção e coordenação do cuidado às pessoas que vivem com HIV/AIDS implicam a elaboração e compartilhamento de um plano de cuidados comum 12,13, de modo que distintas intervenções sejam percebidas e vividas pelos usuários de forma integral, integrada, contínua e adequada às suas necessidades e expectativas 15,43,44, mesmo que em diferentes pontos de atenção à saúde.

Em relação à coordenação da assistência prestada pelas equipes dos SAE, ou seja, a integração horizontal do cuidado encontrou-se que 
os indicadores questionamento de aspectos clínicos durante as consultas, utilização do prontuário médico para a análise da situação de saúde e registro de queixas/percepções, avaliados satisfatoriamente, se relacionam à apropriação de informações para avaliação do estado de saúde, planejamento, operacionalização e reavaliação da assistência 20 .

Contudo, reflexões quanto à qualidade, uso efetivo dos registros e compartilhamento das informações dentro das equipes dos SAE são pertinentes, prevendo dispositivos que favoreçam a integração entre os membros de uma equipe de referência e a incorporação de diretrizes clínicas e protocolos assistenciais que sistematizem o cuidado em consonância com os diferentes perfis de vulnerabilidade dos indivíduos em acompanhamento.

No que se refere a não disponibilização da via impressa dos resultados de exames, a ausência dessa prática pode fragilizar a continuidade e a complementaridade do cuidado prestado em outros pontos de atenção, considerando a utilização de outros serviços/sistemas de saúde pelas pessoas que vivem com HIV/AIDS, podendo gerar duplicidade na realização de exames e custos desnecessários 36 .

Ainda na vertente da integração horizontal do cuidado, a avaliação insatisfatória acerca de questionamentos sobre as condições de vida e recebimento de apoio familiar evidencia o enfoque nas dimensões clínicas/biológicas da doença $6,12,42,45,46$. O descompasso estrutural e organizacional observado na rotina de atendimento dos SAE evidenciado pela desproporção entre o número de profissionais de saúde e de agendamentos/dia interfere no tempo dedicado à abordagem individual das pessoas que vivem com HIV/AIDS, podendo refletir no direcionamento de intervenções clínicas, voltadas principalmente para o manejo da doença em si.

A incorporação de estratégias pautadas na elaboração e operacionalização de instrumentos de classificação das pessoas que vivem com HIV/ AIDS, segundo suas vulnerabilidades individuais e sociais contribuiria para sistematização do cuidado, fortalecendo a coordenação da assistência em suas dimensões horizontal e vertical.

Em relação à coordenação da assistência entre outros serviços de saúde, obteve-se avaliação regular nos encaminhamentos perante as necessidades percebidas pelos usuários e insatisfatória no fornecimento de guias de contrarreferência, quesitos que reforçam a fragmentação do cuidado, divergindo da proposta de uma rede integrada 10 .

Embora o Sistema Hygia possibilite a identificação dos serviços de saúde utilizados pelos usuários, as informações relacionadas à avaliação e respectivas condutas dos profissionais de saúde nem sempre são registradas e atualizadas no sistema, o que requer sensibilização e monitoramento contínuo por parte dos gestores e líderes das equipes 47 . Por outro lado, sabe-se que a disponibilidade de um sistema de informação e a viabilização de rotinas para alimentá-lo, não garante a sua efetiva utilização 48 , exigindo que os profissionais sejam capacitados e motivados constantemente.

No que tange às situações de encaminhamento para outros serviços, embora exista a preocupação dos profissionais dos SAE quanto ao agendamento e obtenção do atendimento, verifica-se que a limitação da autonomia dos usuários quanto à escolha dos serviços de saúde 14 decorre da lógica regionalizada de disponibilização de serviços de referência pré-estabelecida na rede municipal. Contudo, a experiência prévia e grau de satisfação com os serviços designados para o atendimento podem refletir na efetiva utilização e adesão às recomendações terapêuticas 20 , devendo ser explorados pelas equipes no momento do encaminhamento.

De modo geral, a coordenação da assistência às pessoas que vivem com HIV/AIDS foi avaliada satisfatoriamente no município, porém, identificou-se heterogeneidade nos desempenhos entre os SAE.

O SAE “B”, mesmo em processo de organização e estruturação em função da recéminauguração, apresentou um dos melhores desempenhos, possivelmente relacionado à maior disponibilidade de recursos humanos e, consequentemente, maior oferta e flexibilidade de atendimentos. Com destaque também, por sediar a equipe gestora do Programa Municipal de DST/AIDS, aproximando, deste modo, o lócus de decisão, permeado pela dimensão políticogerencial, ao lócus da produção do cuidado, sustentado pelas dimensões técnico-gerenciais 38 .

Transcendendo o enfoque biologicista do agravo, o fato dos SAE "C" e "E" contarem com equipes reduzidas e possuírem interface com instituições de ensino superior pode refletir o descompasso entre a complexidade que envolve o manejo do HIV/AIDS, o trabalho em equipe interdisciplinar 10 e a formação profissional 18,46. Outro elemento é a duplicidade entre as gestões acadêmicas e de saúde, podendo gerar morosidade nos fluxos burocráticos que perpassam os processos decisórios, instabilidade dos recursos disponíveis e prioridades conflitantes, fragilizando a integração entre os membros da equipe (horizontal) e demais pontos de atenção da rede pública municipal (vertical). 
Frente ao exposto, os desafios que envolvem a coordenação da assistência às pessoas que vivem com HIV/AIDS estão relacionados ao estabelecimento de um diálogo permanente entre profissionais dentro e entre os diferentes pontos de atenção 20,21 da rede pública de saúde, envolvendo gestores e usuários, garantindo a legitimidade e sustentabilidade do processo de qualificação e integração do cuidado, em uma perspectiva equânime, integral, contínua e resolutiva.

O menor número de encaminhamentos observado no SAE "A" pode estar relacionado a uma adequada capacidade resolutiva, articulando os recursos disponíveis com as necessidades e demandas identificadas como pela opção de utilização da rede privada de atenção na complementaridade do cuidado.

O SAE “D” não se diferenciou dos demais SAE, não apresentando, portanto, nenhum elemento que o destacasse ou o desvalorizasse perante os outros. Porém, este é um dos mais antigos centros de referência do munícipio, sendo responsável também pelo manejo de outras doenças infecciosas (tuberculose, hanseníase e DST) e pelo atendimento da população carcerária feminina. Possuem suas atividades ao manejo ao HIV/AIDS voltadas para o enfoque clínico dos casos e também apresentam a preocupação da equipe quanto ao contexto de vida e saúde dos parceiros/familiares das pessoas que vivem co HIV/AIDS. Tais achados podem manter relação com a estrutura e capacidade de gestão do serviço e do cuidado, além do próprio aprendizado assistencial em função do tempo de implantação do serviço e experiência quanto ao manejo do agravo e suas comorbidades.

Em decorrência da heterogeneidade na configuração e desempenho dos SAE, dos finitos recursos públicos e das demandas crescentes e complexas relacionadas ao manejo do HIV/AIDS, o êxito na coordenação e manejo deste agravo perpassa por arranjos na dimensão "micro" da rede assistencial, mediante a incorporação de tecnologias inovadoras na produção do cuidado. Na dimensão "meso", por meio da articulação entre equipes dos SAE e demais profissionais, mediado pelas gestões dos serviços e coordenação do Programa Municipal de DST/AIDS. E na dimensão "macro", de modo que as ações de prevenção e manejo do agravo sejam prioridades políticas na agenda de saúde dos gestores.

Reconhece-se como limitações metodológicas desta pesquisa que a análise da coordenação da assistência contemplou apenas a percepção de um grupo de pessoas que vivem com HIV/ AIDS que estava em acompanhamento, estando então, impregnadas por componentes pessoais e subjetivos que envolvem a satisfação com o aten- dimento prestado apenas aqueles sujeitos que foram encaminhamos a outro serviço de saúde. Outra limitação refere-se ao fato de os entrevistados não terem conhecimento das ONGs associadas ao tratamento do HIV/AIDS, não sendo possível avaliar a oferta de orientações sobre as ONGs.

\section{Conclusão}

O presente estudo permitiu delinear o perfil de uma amostra de indivíduos que viviam com AIDS e estavam em acompanhamento nos ambulatórios de referência em HIV/AIDS do Município de Ribeirão Preto. Identificou-se paridade entre os sexos, envelhecimento da população, baixa escolaridade e predomínio da classe econômica C. Quanto às características clínicas, houve predomínio de indivíduos assintomáticos, com boa resposta imunológica e carga viral indetectável, entretanto, verificou-se a presença de comorbidades crônicas tanto de natureza infecciosa como não infecciosa. Tais características e especificidades clínicas, sociais e demográficas permitem refletir sobre a complexidade da assistência a tais indivíduos, de modo à produção do cuidado que englobe uma perspectiva interdisciplinar visando o adequado reconhecimento dos problemas e a garantia de uma coordenação em saúde capaz de compatibilizar a assistência prestada com as necessidades e demandas dos usuários.

Foi possível identificar a complexidade e a fragmentação assistencial no que tange à oferta e continuidade do cuidado prestado às pessoas que vivem com HIV/AIDS em função da utilização de múltiplos serviços de saúde, tanto de natureza pública como privada.

De modo geral, a coordenação da assistência às pessoas que vivem com HIV/AIDS foi avaliada de forma satisfatória no município, entretanto, houve heterogeneidade no desempenho dos cinco SAE, em consonância com as especificidades da estrutura, forma de organização da atenção, atuação profissional, gestão dos serviços de saúde e perfil populacional.

Quanto às potencialidades encontradas no estudo, identificou-se na coordenação da assistência pelos SAE o manejo das condições clínicas diretamente relacionadas à AIDS e, junto a outros serviços a colaboração para obtenção de consultas e o fornecimento de guias de referência. As fragilidades identificadas relacionavam-se ao enfoque biologicista e fragmentado da assistência e falhas na comunicação entre os serviços, reforçando a necessidade do estabelecimento de um trabalho interdisciplinar, incluindo parcerias 
com ONGs e a utilização dos dados contidos nos sistemas de informação, visando assegurar a coerência, continuidade e resolutividade do cuidado prestado no contexto da rede de atenção à saúde.

A constituição e fortalecimento de uma efetiva rede de atenção ao HIV/AIDS, contemplando uma abordagem integral e integrada às pessoas que vivem com HIV/AIDS são fundamentais e exigem sensibilização de diversos atores sociais para o delineamento de um plano de intervenção conjunto, envolvendo equipes e gestores de saú- de, coordenação de programas e representantes dos usuários, garantindo legitimidade ao processo. A utilização de dispositivos formais que favoreçam a integração vertical e horizontal do cuidado é essencial na coordenação da assistência, contudo, há que se refletir sobre outras possíveis lógicas subjacentes que podem permear a integração do cuidado, ou seja, os dispositivos "informais" decorrentes da interação entre os atores envolvidos no processo.

\section{Resumen}

Este estudio tuvo como objetivo analizar la coordinación de acciones y servicios de salud en la asistencia a personas que viven con VIH/SIDA. Se trata de un estudio transversal con 301 personas que viven con VIH/ SIDA. El análisis se realizó mediante técnicas descriptivas, análisis de varianza y comparación múltiple de medias. Se descubrió que la coordinación de la asistencia fue satisfactoria (media $=3,69$ y $S D=1,74$ ). Las personas que viven con el VIH/SIDA cuentan con otros servicios de salud más allá de los Servicios de Apoyo Especializados en VIH/SIDA con los siguientes porcentajes de uso: SAE (atención en urgencias) 67\%; atención primaria 43,2\%; servicios especializados 23,6\%; y privados 15\%). Los cinco SAE evidenciaron diferentes actuacio- nes que se traducen en diversas formas de cuidado, así como en la gestión adecuada del tratamiento clínico en su faceta social. La provisión de una guía de referencia se consideró satisfactoria, incluso frente a la lucha contra elementos insatisfactorios. Es necesario desarrollar estrategias que fomenten el impulso de acciones comunes y coordinadas dentro de los equipos de SAE y entre los diferentes servicios existentes, con el fin de fortalecer decisivamente la atención en la salud dentro de este ámbito.

Síndrome de Inmunodeficiencia Adquirida; Evaluación del Rendimiento de Empleados; Servicios de Salud; Integración de Sistemas 


\section{Colaboradores}

L. M. Lopes trabalhou na concepção, no aperfeiçoamento do instrumento de coleta de dados, no levantamento bibliográfico, na observação e interação com o cenário de pesquisa, no gerenciamento da coleta de dados, na análise, interpretação e discussão dos dados e redação do artigo. G. T. Magnabosco e R. L. P. Andrade participaram na concepção, na coleta, análise, interpretação e discussão dos dados e na redação final do artigo. M. A. Z. Ponce trabalhou na concepção do artigo, na análise, interpretação e discussão dos dados. A. D. Wysocki contribuiu na discussão dos dados, na revisão critica e redação final do artigo. G. M. Ravanholi trabalhou na concepção do artigo, na observação e interação com o cenário de pesquisa, na coleta, análise e discussão dos dados. J. A. Nogueira e T. C. S. Villa contribuíram na concepção do artigo, na definição do quadro teórico e na interpretação e discussão dos dados. I. C. Pinto e R. K. Reis colaboraram na revisão critica e redação final do artigo. L. A. Neves e F. R. A. L. Neves participaram na concepção, na interpretação e na discussão dos dados relacionando com as especificidades do sistema de saúde do município e particularidades de cada Serviço de Assistência Especializada em HIV/AIDS. A. A. Monroe trabalhou na concepção, na definição do quadro teórico, na analise, interpretação e discussão dos dados, na revisão critica e redação final do artigo. L. M. Scatena participou do processo de revisão e readequação metodológica da nova versão do manuscrito apresentada, em especial no que tange aos procedimentos de análise dos dados, resultados e discussão dos mesmos.

\section{Agradecimentos}

O estudo teve financiamento da FAPESP (processo: 2010/17607-7), da Capes e do ICOMRTA AIDS/TBInternational Clinical, Oerational, and Health Services Research and Training Award; Center for TB Research; colaboração e participação da equipe do Programa Municipal de DST/AIDS e Hepatites Virais.

\section{Referências}

1. Berquó E, Barbosa MR. Introdução: a epidemia de HIV/Aids apresenta-se como das mais complexas e desafiadoras pandemias já enfrentadas pela humanidade. Rev Saúde Pública 2008; 42 Suppl 1:7-11.

2. Brito AM, Castilho EA, Szwarcwald CL. AIDS e in fecção pelo HIV no Brasil: uma epidemia multifacetada. Rev Soc Bras Med Trop 2000; 34:207-17.

3. Pereira AJ, Nichiata LYI. A sociedade civil contra a AIDS: demandas coletivas e políticas públicas. Ciênc Saúde Coletiva 2011; 16:3249-57.
4. Moraes DR. Revistando as concepções de integralidade. Rev APS 2006; 9:64-72.

5. Conti S, Masocco M, Pezzotti P, Toccaceli V, Vichi M, Boros S, et al. Differential impact of combined antiretroviral therapy on the survival of Italian patients with specific AIDS-defining illness. J Acquir Immune Defic Syndr 2000; 25:451-8.

6. Schaurich D, Coelho DF, Motta MGC. A cronicidade no processo saúde-doença: repensando a epidemia da AIDS após os anti-retrovirais. Rev Enferm UERJ 2006; 14:455-62. 
7. United Nations Programme on HIV/AIDS. Together we will end AIDS. Geneva: United Nations Programme on HIV/AIDS; 2012.

8. Lavor AD. A epidemia não acabou. Radis Comunicação e Saúde 2012; (123):9-15.

9. Departamento de DST, Aids e Hepatites Virais, Secretaria de Vigilância em Saúde, Ministério da Saúde. Boletim Epidemiológico Aids e DST 2011; 8(1).

10. Borges MJL, Sampaio AS, Gurgel IGD. Trabalho em equipe e interdisciplinaridade: desafios para a efetivação da integralidade na assistência ambulatorial às pessoas vivendo com o HIV/AIDS em Pernambuco. Ciênc Saúde Coletiva 2012; 17:147-56.

11. Departamento de Atenção Básica, Secretaria de Atenção à Saúde, Ministério da Saúde. HIV/AIDS, hepatites e outras DST. Brasília: Ministério da Saúde; 2006. (Cadernos de Atenção Básica, 18).

12. Nemes MIB, Castanheira ERL, Melchior R, Alves MTSSB, Basso CR. Avaliação da qualidade da assistência no programa de AIDS: questões para a investigação de serviços em saúde no Brasil. Cad Saúde Pública 2004; 20 Suppl 2:S310-21.

13. Pinheiro R. A importância do SUS: o político, o social e as questões a superar. In: Raxach JC, organizador. Reflexões sobre assistência à AIDS: relação médico-paciente, interdisciplinaridade e integralidade. Rio de Janeiro: Associação Brasileira Interdiciplinar de AIDS; 2003. p. 59-61.

14. Palacio MB, Figueiredo MAC, Souza LB. O cuidado em HIV/AIDS e a atenção primária em saúde: possibilidades de integração da assistência. Psico (Porto Alegre) 2012; 43:360-7.

15. Hartz ZMA, Contandriopoulos A-P. Integralidade da atenção e integração de serviços de saúde: desafios para avaliar a implantação de um "sistema sem muros”. Cad Saúde Pública 2004; 20 Suppl 2:S331-6.

16. Vasconcelos CM, Pasche DF. O Sistema Único de Saúde. In: Campos GWS, Minayo MCS, Akerman M, Drumond Jr. M, Carvalho YM, organizadores. Tratado de saúde coletiva. São Paulo: Editora Hucitec/Rio de Janeiro: Editora Fiocruz; 2006. p. 53162. (Saúde em Debate, 170).

17. Melchior R, Nemes MIB, Basso CR, Castanheira ERL, Alves MTSB, Buchalla CM, et al. Avaliação da estrutura organizacional da assistência ambulatorial em HIV/Aids no Brasil. Rev Saúde Pública 2006; 4:143-51.

18. Nemes MI, Castanheira ER, Helena ET, Melchior R, Caraciolo JM, Basso CR, et al. Treatment adherence, access and AIDS assistance quality in Brazil. Rev Assoc Med Bras 2009; 55:207-12.

19. Sullivan PS, Denniston M, Mokotoff E, Buskin S, Broyles S, McNaghten AD. Quality of care for HIV infection provided by Ryan White Program-supported versus Non-Ryan White Program-supported facilities. PLoS One 2008; 3:e3250.

20. Starfield B. Atenção primária: equilíbrio entre necessidades de saúde, serviços e tecnologia. Brasília: Organização das Nações Unidas para a Educação, a Ciência e a Cultura; 2002.

21. Kringos DS, Boerma WGW, Hutchinson A, Zee J, Groenewegen PP. The breadth of primary care: a systematic literature review of its core dimensions. BMC Health Serv Res 2010; 10:65.
22. Programa Nacional de DST e Aids, Secretaria de Vigilância em Saúde, Ministério da Saúde. Recomendações para terapia anti-retroviral em adultos infectados pelo HIV. Brasília: Ministério da Saúde; 2008.

23. Fundação Hospitalar do Estado de Minas Gerais. Protocolo de acompanhamento ambulatorial do paciente com HIV/AIDS. Belo Horizonte: Fundação Hospitalar do Estado de Minas Gerais; 2009.

24. Almeida CM, Macinko J. Validação de uma metodologia de avaliação rápida das características organizacionais e do desempenho dos serviços de atenção básica do Sistema Único de Saúde (SUS) em nível local. Brasília: Organização Pan-Americana da Saúde; 2006.

25. Pasquali L. Instrumentos psicológicos: manual prático de elaboração. Instituto de Psicologia. Brasília: Universidade de Brasília/Laboratório de Pesquisa em Avaliação e Medida, Instituto Brasileiro de Avaliação e Pesquisa em Psicologia; 1999.

26. Nemes MIB, Bassos CR, Castanheira ERL, Melchior R, Alencar TMD, Caraciolo JMM, et al. Qualiaids: avaliação e monitoramento da qualidade da assistência ambulatorial em AIDS no SUS. Brasília: Ministério da Saúde; 2008.

27. Maroco J. Análise estatística com utilização do SPSS. 3a Ed. Lisboa: Edições Sílabo; 2007.

28. Santos NJS, Buchalla CM, Filipe EV, Bugamelli L, Garcia S, Paiva V. Mulheres HIV positivas, reprodução e sexualidade. Rev Saúde Pública 2002; 36 Suppl:12-23.

29. Pottes FA, Brito AM, Gouveia GC, Araújo EC, Carneiro RM. Aids e envelhecimento: características dos casos com idade igual ou maior que 50 anos em Pernambuco, de 1990 a 2000. Rev Bras Epidemiol 2007; 10:338-51.

30. Pinto ACS, Pinheiro PNC, Vieira NFC, Alves MDS. Compreensão da pandemia da AIDS nos últimos 25 anos. DST J Bras Doenças Sex Transm 2007; 19:45-50.

31. Souza Z, Leite JL. Aids e envelhecimento: reflexões sobre a infecção pelo HIV em indivíduos acima dos 60 anos. http://www.saude.rio.rj.gov.br/ saude/pubsms/media/aidsenvelhecimento.doc (acessado em 16/Mai/2010).

32. Camargo BV, Bertoldo RB. Comparação da vulnerabilidade de estudantes da escola pública e particular em relação ao HIV. Estud Psicol (Campinas) 2006; 23:369-79.

33. Girondi JBR, Zanatta AB, Bastiani JAN, Nothaft SS, Santos SM. Perfil epidemiológico de idosos brasileiros que morreram por síndrome da imunodeficiência adquirida entre 1996 e 2007. Acta Paul Enferm 2012; 25:302-7.

34. Silva EFR, Lewi DS, Tenore SB, Bassichetto KC. Estado nutricional clínico e padrão alimentar de pessoas vivendo com HIV/AIDS em assistência ambulatorial no município de São Paulo. Rev Bras Epidemiol 2010; 13:1-12.

35. Fernandes LCL, Bertoldi AD, Barros AJD. Utilização dos serviços de saúde pela população coberta pela Estratégia de Saúde da Família. Rev Saúde Pública 2009; 43:595-603. 
36. Mendes EV. O cuidado das condições crônicas na atenção primária à saúde: o imperativo da consolidação da estratégia da saúde da família. Brasília: Organização Pan-Americana da Saúde; 2012.

37. Gomide MFS, Pinto IC, Gomide DMP, Zacharias FCM. Perfil de usuários em um serviço de prontoatendimento. Medicina (Ribeirão Preto) 2012; 45:31-8.

38. Monroe AA, Gonzales RIC, Palha PF, Sassaki CM, Ruffino-Netto A, Vendramini SHF, et al. Envolvimento de equipes da atenção básica à saúde no controle da tuberculose. Rev Esc Enferm USP 2008; 42:262-7.

39. Sadala MLA, Marques AS. Vinte anos de assistência a pessoas vivendo com HIV/AIDS no Brasil: a perspectiva de profissionais da saúde. Cad Saúde Pública 2006; 22:2369-78.

40. Ferraz DAS, Nemes MIB. Avaliação da implantação de atividades de prevenção das DST/AIDS na atenção básica: um estudo de caso na Região Metropolitana de São Paulo, Brasil. Cad Saúde Pública 2009; 25 Suppl 2:S240-50.

41. Contandriopoulos A-P, Denis J-L, Touati N, Rodriguez R. Intégration des soins: dimensions etmiseen-oeuvre. Ruptures 2001; 8:38-52.

42. Oliveira IBN. Acesso universal? Obstáculos ao acesso, continuidade do uso e gênero em um serviço especializado em HIV/AIDS em Salvador, Bahia, Brasil. Cad Saúde Pública 2009; 25 Suppl 2:S259-68
43. Hofmarcher MM, Oxley H, Rusticelli E. Improved health system performance through better care coordination. Paris: Organisation for Economic Co-operation and Development; 2007.

44. Martínez DH, Navarrete ML, Lorenzo IV. Factores que influyen en la coordinación entre niveles asistenciales según la opinión de directivos y profesionales sanitarios. Gac Sanit 2009; 23:280-6.

45. Santos SMJ, Nóbrega MMLN. Ações de enfermagem identificadas no Projeto CIPESC e utilizadas no cuidado de pacientes com Aids. Rev Esc Enferm USP 2004; 38:369-78.

46. Sarna A, Kellerman S. Access to antiretroviral therapy for adults and children with HIV infection in developing countries: Horizons Studies, 20022008. Public Health Rep 2010; 125:305-15.

47. Cruz MM, Toledo LM, Santos EM. O sistema de informação de AIDS do Município do Rio de Janeiro: suas limitações e potencialidades enquanto instrumento da vigilância epidemiológica. Cad Saúde Pública 2003; 19:81-9.

48. Almeida PF, Giovanella L, Mendonça MHM, Escorel S. Desafios à coordenação dos cuidados em saúde: estratégias de integração entre níveis assistenciais em grandes centros urbanos. Cad Saúde Pública 2010; 26:286-98.

Recebido em 25/Abr/2013

Versão final reapresentada em 01/Abr/2014

Aprovado em 05/Mai/2014 